\section{NERC corporate plan}

SIR - Your news item (Nature 10 January, p.91) on the corporate plan for the reorganization of the Natural Environment Research Council (NERC) is misleading. It implies that trades unions are now being consulted about the plan but many staff, certainly those scientists that the Institution of Professional Civil Servants represents in the British Geological Survey, regard the council's consultations with its unions as a sham. Most of the staff have not yet been allowed to see the document and they are anxious at the reported implications and angry at being refused sight of it. The few departmental union representatives who are privy to the plan are forbidden to discuss it with their members.

NERC has been reported as saying that the reason for the secrecy is fear of misunderstanding but secrecy is far more likely to foster misunderstanding. As there is already widespread opposition to the plan, many believe the secrecy is really designed to minimize dissent before the plan is implemented.

NeIL AITKENHEAD

British Geological Survey Section,

(Chairman)

Institution of Professional Civil

Servants (NERC Branch),

Keyworth,

Nottingham NG12 5GG, UK

\title{
US space station experiments
}

SIR - In his article opposing European participation in the US space station project (Nature 1 November, p.11), Dr Erhard Keppler unduly minimizes the potential for biological investigation on board the proposed facility with his statement that interest in such work "... has greatly lost its attraction in the past few years". While it is true that fewer studies on the basic biological consequences of microgravity have recently appeared in the literature than one might expect, I believe that this is largely owing to a lack of proper facilities rather than to a shortage of interest.

An examination of earlier work published on vertebrate development in space reveals a depressing parade of experiments marred by lack of controls, launch delays, short flight times and constraints on cabin space and crew involvement, resulting in data of limited value. For example, the Soviets $^{1}$ flew developing frog larvae on board the Salyut- 4 spacecraft and found changes that could be interpreted as degenerative in the vestibular sensory neuroepithelium of the inner ear, but as in the case of most other orbital biology work so far performed, $1 \mathrm{~g}$ control centrifuge data are missing, so drawing a conclusion from the findings is risky. Similar experiments flown on Gemini ${ }^{2}$ and Biosatellite ${ }^{3}$ flights also yielded unsatisfactory results. Good information on microgravitational effects on higher vertebrate development is especially scarce. A joint US-Soviet attempt to mate rats in orbit on Cosmos $1129^{4}$ failed to produce conception for reasons that are not clear (behaviour was apparently not recorded), while 60 incubating quail eggs on the same flight suffered developmental arrest when the humidifier broke down in mid-flight.

Rather than throwing up our hands and giving up, we should insist on proper facilities where careful long-term observations can be made directly by specialists. While the NASA/ESA Spacelab is a giant step in this direction (with some planned flights of the orbital laboratory devoted specifically to the life sciences), only a per- manent space station offers the opportunity for continuing studies of animals and plants reproducing and developing in microgravity over multiple generations. This work has extremely important implications for the future of man in space and constitutes strong justification for going forward with the construction of a space station. Cooperation with European bioscience groups in such an endeavour would clearly benefit all. RUSSEL L. KERSCHMANN Massachusetts General Hospital,

Boston, Massachusetts 02114, USA

1. Vinnikov, Y.A. et al. Arkh. Anat. Gistol, Embryrol. 70 no.1 (NASA TT F-16987, 1976).

2. Young, R.S. \& Tremor, J.W. Life Sci. Space Res. 6, 87 (1968).

Edwards, B.F. Life Sci. Space Res. 7, 84 (1969)

4. Keefe, J.R. in Final Reports of US Rat Experiments Flown on the Soviet Satellite Cosmos 1129 (NASA TM-81298, (1979).

\section{Nuclear winter}

SIR - The National' Research Council of the US Academy of Sciences has now joined others who have postulated severe winter conditions as an immediate consequence of any major nuclear war. So far such hypotheses have suggested that such conditions may be of limited duration a matter of weeks or months - and appear to have concentrated on the direct loss of solar energy that would result from pollution of the atmosphere.

It would therefore be of interest to know whether any of the models so far used have taken account of predictable consequential energy losses - notably those attributable to the albedo effect of the snowfields that the nuclear winter would create and those that might result from the interruption of warm air and tidal flows into the higher latitudes of the Northern Hemisphere if the seas froze. On basic principles, it seems entirely possible that the aggregate of effects might well tip the scales to precipitate a major glaciation.

Rhyd yr Harding,

Castle Morris,

near Haverfordwest SA62 SEJ, UK
Jerne still influential

SIR - Peter Newmark's use of the past tense in his brief review of Niels Jerne's Nobel Prize winning work (Nature 311, $601 ; 1984)$ is misleading. He writes that Jerne's network theory "was at its most influential about a decade ago". This seems to imply that the theory has since been discredited, and is no longer influential. It was however only a decade ago that Jerne's most famous network paper was published in the Annales d'Immunologie (Institut Pasteur), and it took some time for the concept to become popular. One measure of the degree to which a paper is influential is the extent to which it is cited. Figure 1 shows the number of citations of the abovementioned paper for each year since then, according to the Science Citation Index. To judge by this criterion, that paper's degree of influence may not yet have peaked.

It is impractical to attempt to review in this letter the very considerable achievements of immune system network theory, as formulated by Jerne and subsequently modified and developed in more detail by others. Suffice it to say that Newmark's remarks clearly do not reflect current thinking of workers in the field.

GEOFFREY W. HOFFMANN Departments of Physics and Microbiology, University of British Columbia,

Vancouver, BC, Canada

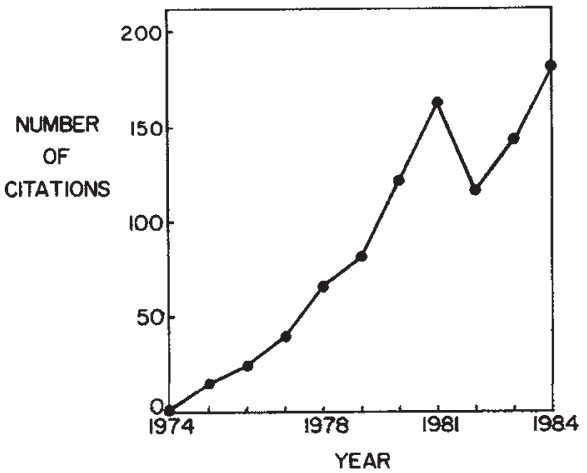

The number of citations of the paper "Toward a Network Theory of the Immune System" by N.K. Jerne, Annls Immun., Inst. Pasteur, Paris 125C, 373-389 (1974) for the years 1974-84. The number given for 1984 is twice the number of citations for January to June 1984.

\section{Value-free science}

SIR - Your correspondent Mark Diensdorf (Nature 10 January, p.92) throws at us the same superstitious rubbish we have been enduring for years. Is he (or anyone else) really unable to distinguish between the scientific (and value-free) statement "The lethal dose of cyanide is $x$ $\mathrm{gm} /$ kilo of body weight" and the practical (and value-loaded) statement "I am going to poison my wife'?

M. HAMMERTON University of Newcastle upon Tyne, Department of Psychology,

Ridley Building, Claremont Place, Newcastle upon Tyne NEI 7RU, UK 